Estimating the Reliability of Emotion Measures over Very Short Intervals: The Utility of WithinSession Retest Correlations

Graham H. Lowman

University of Alabama

Dustin Wood

University of Alabama

Benjamin F. Armstrong III

McGill University

P.D. Harms

University of Alabama

David Watson

University of Notre Dame

Correspondence should be sent to Graham Lowman, Department of Management, University of Alabama, 361 Stadium Dr., Tuscaloosa, AL 35487-0225.

Contact: ghlowman@crimson.ua.edu 


\begin{abstract}
Short measures are commonly used when conducting research involving emotions. However, obtaining appropriate estimates of reliability for short measures is traditionally problematic and is a reoccurring concern in emotion research. To address this issue, we compare the withinsession test-retest and factor analysis methods for estimating the reliability of items in the PANAS-X. Results indicate that within-session test-retest $\left(r_{X(d)}\right)$ estimates outperform the factor analysis method by demonstrating stronger relationships with item properties relevant to reliability and validity-related criteria. In addition, $r_{X(d)}$ estimates appropriately generalize across samples with various instruction stems and prevent corrections for attenuation greater than 1.00. Therefore, we encourage researchers to use the corresponding average item-level $r_{X(d)}$ estimates reported here to correct for attenuation when examining single-items from the PANAS$\mathrm{X}$ if a test-retest design is not feasible.
\end{abstract}

Keywords: single-item reliability estimates over short time intervals; PANAS-X; test-retest reliability; estimates for appropriate correction for attenuation 


\section{Estimating the Reliability of Emotion Measures over Very Short Intervals: The Utility of Within-Session Retest Correlations}

In this study, we illustrate how to properly estimate the reliability of single-item measures of emotion over short intervals. Although single-item measures are common within the emotion literature (Weidman, Steckler, \& Tracy, 2017), it has been difficult to determine how to estimate their reliability. This is because reliability is often estimated using internal consistency statistics such as coefficient alpha (Cronbach, 1951), which are usually understood to require multi-item scales (Nunnally \& Bernstein, 1991; though see Wanous \& Hudy, 2001). Compounding this, internal consistency statistics are known to (1) systematically underestimate the reliability of the measure over the measurement interval, due to reliable item-specific variance (Cronbach, 1951; Guttman, 1945; John \& Soto, 2007) and (2) do so by different magnitudes across scales in ways that are difficult to index.

The lack of appropriate reliability estimates for single-item emotion measures leaves researchers with the unenviable option of either (1) using systemically underestimated reliability estimates, resulting in associations between variables that are overestimated when these estimates are used to adjust for unreliability (e.g., Osburn, 2000), or (2) simply not adjusting for unreliability, causing associations to be systematically underestimated.

\section{Within-Session Retest Correlations}

A potential solution to this problem is the use of within-session retest correlations, or $r_{X(d)}$ estimates, where the same items are assessed twice within a survey after a short retest interval $d$ (e.g, 10 to 15 minutes) in which participants complete other measures or tasks. ${ }^{1}$ Early psychometric concerns about immediately retesting measures to estimate reliability largely involved a participant's ability to recall previous answers (Cronbach, 1947, 1951; Guttman, 1945). Therefore, the retest interval $d$ is purposefully selected to reduce the ability of participants 
to remember their specific answers in the first assessment by (1) introducing an amount of material between assessments that exceeds an individual's working memory limits and (2) randomizing the ordering of items within the assessments (Alvarez \& Cavanagh, 2004).

As detailed by Wood et al. (2017), $r_{X(d)}$ values have many advantages as reliability estimates. Perhaps most importantly, they operationalize reliability coefficients as the degree of reproducibility of scores on the same test over repeated assessments, which more directly operationalizes reliability to fit its conceptual meaning as "the correlation of a measure with itself” (John \& Soto, 2007; p. 464; Guttman, 1945). They also can be estimated on scales of any length, including single-item measures. Additionally, $r_{X(d)}$ estimates have shown advantageous properties as reliability estimates when examined empirically, as they (1) provide fairly consistent estimates across samples, (2) tend to be consistently larger in magnitude than coefficient alpha, and (3) better track inter-item variation in validity-related criteria than coefficient alpha (de Vries, Realo, \& Allik, 2016; McCrae, Kurtz, Yamagata, \& Terracciano, 2011). Improved tracking is due to how validity criteria such as self-other agreement and longterm stability are operationalized. Specifically, these are estimated as correlations involving the same items administered in different ways (e.g., by self-ratings and by peer-ratings). ${ }^{2}$ In contrast, internal consistency statistics such as coefficient alpha are affected by score consistency and the degree of heterogeneity of content within the scale, which is often arbitrarily determined (e.g., whether to create a 'broad' scale of positive affectivity, or a 'narrow' scale of joy). This introduces another source of variance affecting internal consistency statistics irrelevant of the extent to which participants provide reliable scores.

In the current study, we use a very common measure in emotion research, the Positive and Negative Affect Schedule - Expanded Form (PANAS-X; Watson \& Clark, 1999), to explore 
whether within-session retest correlations show these properties for emotion measures as well. We examined several questions:

1) Are $r_{X(d)}$ estimates consistent across samples?

2) Are $r_{X(d)}$ estimates consistently higher than internal consistency estimates?

3) Are $r_{X(d)}$ estimates more predictive of validity-related criteria (e.g., self-other agreement), and with item properties known to affect reliability (e.g., score variability)?

\section{Method}

\section{Samples for Obtaining Within-Sample Retest Correlations}

Within-session MTurk Sample 1 (MT1). Participants were recruited using Amazon's Mechanical Turk (MTurk); 48\% were female $\left(M_{\mathrm{age}}=34.8\right.$ years; $\left.S D=11.31\right)$. An initial 213 individuals completed the survey. Following recommendations by Wood, Harms, Lowman, and Desimone (2017), participants were removed from the analysis if they completed the PANAS-X at a rate faster than 1 -second per item. Also accounting for missing data, this resulted in a final sample ranging from 173 to 177 . In all samples, the PANAS-X items were rated on a 5-point scale (1=very slightly or not at all to 5=extremely). In this sample, participants rated the items under the instruction: "Indicate to what extent you are feeling like this in general". The average time between the PANAS-X administrations within the session was approximately 10 minutes.

Within-session MTurk Sample 2 (MT2). Participants were part of a larger study in which they completed other measures involving workplace behaviors not considered here (62\% male; $M_{\text {age }}=34$ years; $S D=10.1$ ). An initial 142 participants completed the portion of the survey used in this study. After accounting for missing data and removing respondents who completed this section of the survey faster than 1 second per item, the final sample sizes ranged from 120 to 107 for each item. The instruction stem was: "Indicate to what extent you have felt this way 
during the past few weeks". The average time between PANAS-X administrations within the session was approximately 12 minutes.

Multi-session Student Sample 1 (SS). The sample consisted of undergraduate students (65\% female; $M_{\text {age }}=23$ years; $\left.S D=4.56\right) ; 90$ students initially completed the survey. Participants were removed from the analysis if they completed either PANAS-X administration in under 1 second per item. After accounting for missing data, this resulted in a final sample of 82 to 85 for each item. The instruction stem was: "Indicate to what extent you have felt this way today". The average time between within-session administrations was approximately 20-25 minutes. Eightyfive participants also completed a third administration approximately one week later.

\section{Samples for Validity Criteria}

Multi-session Young Adult Sample (YA). Data from the Iowa Longitudinal Personality Project (ILPP) provided test-retest reliability estimates over longer time periods. This data set has been examined in prior studies (Vaidya, Gray, Haig, Mroczek, \& Watson, 2008; Vaidya, Gray, Haig, \& Watson, 2002), and consists of five assessments spaced roughly three years apart. At the first (1996) and second (1999) assessments, 398 undergraduate students completed the PANAS-X (76\% female; $M_{\text {age }}=21.1$ years). At Time 3 (2002), 310 of the original participants completed the assessment. The Time 4 (2005) and Time 5 (2009) assessments were completed by 270 and 279 participants, respectively. The instruction stem was: "To what extent you generally feel this way, that is, how you feel on average?".

Self-other Agreement Samples (SO). We used three samples of self-other agreement ratings that were reported by Watson, Hubbard, and Wiese (2000). The first sample consisted of 74 married couples $\left(M_{\mathrm{age}}=47\right.$ years; $M_{\text {relationship }}=202.6$ months $) .{ }^{3}$ The dating sample consisted of 136 heterosexual couples $\left(M_{\text {age }}=18.2\right.$ months; $M_{\text {relationship }}=36$ months $)$. The third sample consisted 


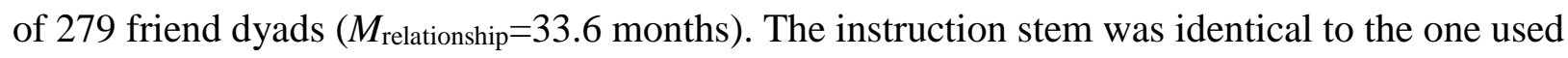
in the young adult sample.

Given the number of respondents who were removed using the quality control measures, we conducted analyses with and without these controls. When fast respondents $(<1-$ spi) were included in the analysis, the relationships among $r_{X(d)}, \widetilde{I C}_{x}$, and validated-related criteria generally decreased (see Supplementary Materials). Approval to conduct the proposed analyses was received from the University of Alabama institutional review board.

\section{Results}

\section{Properties of Average Magnitude of Scale-Level Reliability Estimates}

We first compared properties of $r_{X(d)}$ estimates to alpha estimates at the scale-level. Estimates for $r_{X(d)}$ were calculated at the scale-level by first aggregating items for each scale and then correlating within-session participant ratings for the 11 scales. Additional properties of the PANAS-X scales are given in the Supplementary Materials.

When averaged across the three within-session samples, alpha reliability estimates for the first administration at the scale-level ranged from .76 (Surprise) to .94 (Joviality), with a mean of .85. In comparison, the three-sample average $r_{X(d)}$ estimates ranged from .74 (Hostility) to .91 (Self-Assurance and Joviality), with an average magnitude of .84. Thus, in contrast to earlier comparisons of these two statistics (Wood et al., 2017), $r_{X(d)}$ estimates were not systematically higher than alpha estimates within the PANAS-X.

\section{Properties of Item-Level Reliability Estimates}

Item-level $r_{X(d)}$ estimates were calculated by correlating the first and second ratings across participants for each of the 60 items. For comparison purposes, we also computed approximate internal-consistency estimates of the reliability of single items using a factor 
analytic approach (see Denissen, Geenen, Selfhout, \& Van Aken, 2008; Fisher, Matthews, \& Gibbons, 2015; Spörrle \& Bekk, 2014; Wanous \& Hudy, 2001; Weiss, 1976), which we denote as $\widetilde{I C}_{x}$. In this approach, all items within a scale are loaded on a single factor via principal axis factoring. The item's estimated communality (i.e., squared factor loading) then serves to indicate how well the item would correlate with a 'parallel form' of itself, assuming that the factor serves as the only expected source of score consistency. Because this approach assumes items are unidimensional, only the 55 items placed on the 11 specific affect scales were included in this analysis (i.e., we did not include the five items that are scored only on the general Negative Affect and Positive Affect scales).

Consistency of estimates across samples. The rank-order correlations for the internalconsistency estimates were positive and significant across samples $(q=.38$ to $.47, p<.01) .{ }^{4}$ The rank-order correlations for the item-level $r_{X(d)}$ estimates were also positive and significant, specifically $\mathrm{MT}_{1}$ and $\mathrm{MT}_{2} q=.45, p<.01, \mathrm{MT}_{1}$ and SS $q=.48, p<.01$, and SS and $\mathrm{MT}_{2} q=.30$, $p=.02$.

\section{Item Properties and Validity-Related Criteria}

To compare the two approaches, average item-level test-retest $\left(\bar{r}_{X(d)}\right)$ and approximate internal-consistency $\left(\widetilde{I C}_{x}\right)$ estimates were correlated with item properties relevant to reliability and validity-related criteria (Table 2). An item's deviation from the scale midpoint (estimated as $\left.\left|M_{X}-3\right|\right)$ is expected to be associated with decreased standard deviation estimates for the item, which in turn is expected to decrease an item's reliability due to range restriction or ceiling/floor effects (e.g., Wood \& Wortman, 2012). As expected, item-level $\bar{r}_{X(d)}$ was strongly associated with item means $(q=.35 ; p<.01)$ and item deviation from the scale midpoint $(q=-.46 ; p<.01)$. Additionally, $\bar{r}_{X(d)}$ was strongly correlated with item standard deviation $(q=.60 ; p<.01)$. 
The $\bar{r}_{X(d)}$ estimates also were generally associated with validity-related criteria. In terms of stability, $\bar{r}_{X(d)}$ was significantly correlated with 1-week and 3-year stability estimates ( $q s \geq .29$; $p s<.05)$. Additionally, $\bar{r}_{X(d)}$ was significantly related to self-other agreement in both dating and friend relationships ( $q \geq .30 ; p s<.05)$. In comparison, $\widetilde{I C}_{x}$ estimates were not significantly positively associated with any stability correlations (all $q s<.16$ ), with the exception of the married self-other agreement correlations.

Overall, $\bar{r}_{X(d)}$ estimates were consistently more positively associated with reliabilityand validity-related criteria than were $\widetilde{I C}_{X}$ estimates, and many of these differences were statistically significant. Together, these results indicate the value of $r_{X(d)}$ as reliability estimates.

\section{Discussion}

Appropriately estimating the reliability of single-item measures is a recurring issue in emotion research (Weidman et al., 2017). To address this issue, we explored properties of within-session retest correlations, or $r_{X(d)}$ estimates, as reliability estimates. In contrast to prior examinations, which found $r_{X(d)}$ estimates to be larger than traditional alpha estimates for the Big Five Inventory-2 (BFI-2; Soto \& John, 2016; Wood et al., 2017), PANAS-X scale-level $r_{X(d)}$ and alpha estimates were comparable in magnitude. This is likely due to the PANAS-X scales having a greater degree of content similarity across items within a given scale than the BFI-2 (Gulliksen, 1945; McCrae et al., 2011). This is demonstrated by the PANAS-X scales having considerably higher average inter-item correlations $(M=.63)$ than those found for the BFI$2(M=.47)$ in the $\mathrm{MT}_{2}$ Sample. Paired with the findings from Wood et al. $(2017)^{5}$, this suggests that traditional internal consistency statistics better approximate $r_{X(d)}$ estimates when scales contain more redundant items. 
However, the correlates of $r_{X(d)}$ at the item-level suggest that within-session retest correlations offer many advantages as reliability estimates (de Vries et al., 2016). Specifically, they provided a rank-ordering of $r_{X(d)}$ estimates that was consistent across different samples and more closely tracked item properties associated with reliability and validity related criteria (McCrae et al., 2011; Wood \& Wortman, 2012) than internal consistency statistics. In comparison, $\widetilde{I C}_{x}$ estimates underperformed relative to $r_{X(d)}$ estimates, as they failed to correlate appropriately with item properties and estimates of validity-related criteria and resulted in nonsensical corrections for attenuation that were greater than $1.00 .^{6}$

Based on these results, $\bar{r}_{X(d)}$ estimates reported for the PANAS-X are useful estimates of reliability and provide more valid estimates than internal consistency values. These conclusions are strengthened by the supported generalizability across samples and instruction sets. Therefore, if a test-retest design is not feasible, we recommend that researchers use the corresponding itemlevel $\bar{r}_{X(d)}$ estimates reported here to correct for attenuation when examining single-items from the PANAS-X. Our results indicate that this approach provides considerably more valid estimates of reliability than item-level internal-consistency estimates, which are regularly prescribed as a 'best practice' for estimating item-level reliabilities (Spörrle \& Bekk, 2014; Wanous \& Hudy, 2001; Weiss, 1976).

Given daily diary or experience sampling studies (e.g., Bjälkebring et al., 2015; Chin et al., 2016) often require single-item measures, $\bar{r}_{X(d)}$ estimates could also be used to correct for attenuation when assessing emotional states. The consistency in the item-level rank-order correlations across samples with different instructions sets-ranging from emotions felt "in general" (MT 1$)$ to "today" $(\mathrm{SS})$ — suggests that the $\bar{r}_{X(d)}$ estimates reported here likely generalize 
to instruction sets aimed at assessing emotional states (e.g., how I feel "right now"). However, future studies are needed to confirm this generalization.

As with all short-term retest studies, one potential concern is a lack of sufficient independence between assessments due to memory effects artificially enhancing response consistency. To address this concern, we included a large number of questions between PANAS$\mathrm{X}$ administrations in the $\mathrm{MT}_{2}$ sample. Given working memory is generally limited to 3 or 4 pieces of information (Alvarez \& Cavanagh, 2004) and item order within the survey was randomized, it is unlikely memory effects influenced responses on the second assessment. This is also supported by consistency in the rank-order of $r_{X(d)}$ estimates across samples; therefore, independence likely was maintained in each of the retest samples. A related concern is mood changes due to participants reacting to questions between assessments. However, similar to memory effects, the consistency of $r_{X(d)}$ estimates across samples using various items between assessments suggests the influence of any particular item on the results is limited.

Future studies should continue to test the utility of within-session retest correlations as estimates of the reliability of single-item emotion measures. Although concerns still exist regarding the use of single-item measures (e.g., Boyd et al., 2005), researchers and practitioners interested in emotion and emotional states often use these measures for their efficiency in capturing participants' emotions. The test-retest approach provides valid single-item reliability estimates, and shows that they are surprisingly high. For instance, the average $\bar{r}_{X(d)}$ estimate in the current study was .71, higher than we imagine most researchers would expect. This, in turn, addresses one of the major concerns regarding single-item measures and enables researchers and practitioners to evaluate the true relationships between emotion constructs more accurately. 


\section{Footnotes}

${ }^{1} r_{X(d)}$ indicates the correlation of $\mathrm{X}$ with itself when rated twice in different administrations of the same inventory; the interval $d$ is defined by both the length of time and the number and type of items separating the repeated inventory.

${ }^{2}$ Specifically, a measure's correlations with other variables should not exceed the square root of the measure's correlation with itself (i.e., its reliability); $\rho_{X Y} \leq \sqrt{\rho_{X X}}$ (Lord \& Novick, 1968).

${ }^{3} M_{\text {relationship }}$ indicates mean length of relationship (i.e., number of months married, number of months dating, and number of months friends).

${ }^{4}$ Following Cattell (1952), $q$ indicates correlations at the between-item level (thereby reserving $r$ for between-person correlations).

${ }^{5}$ Results from Wood et al. (2017) indicated $r_{X(d)}$ estimates ranged from .82 to $.95(M=.89)$ and tradition alpha estimates ranged from .74 to $.84(M=.83)$ for the BFI-2. See Table 9 in the supplementary materials for scale-level average inter-item correlations for the PANAS-X and $\mathrm{BFI}-2$ collected in the $\mathrm{MT}_{2}$ Sample.

${ }^{6}$ The test-retest correlations for YA and SO samples were corrected for attenuation by dividing the correlations by the corresponding item-level $\bar{r}_{X(d)}$ and $\widetilde{I C}_{x}$ estimates. This resulted in 600 corrections for attenuation using $\bar{r}_{X(d)}$ estimates, with zero corrections exceeding a value of 1.00; and 550 corrections for attenuation using the $\widetilde{I C}_{x}$ estimates, with 18 corrections exceeding a value of 1.00 . 


\section{References}

Alvarez, G. A., \& Cavanagh, P. (2004). The capacity of visual short-term memory is set both by visual information load and by number of objects. Psychological Science, 15(2), 106-111.

Bjälkebring, P., Västfjäll, D., Svenson, O., \& Slovic, P. (2015). Regulation of experienced and anticipated regret in daily decision making. Emotion, 16(3), 381-386.

Boyd, B. K., Gove, S., \& Hitt, M. A. (2005). Construct measurement in strategic management research: Illusion or reality? Strategic Management Journal, 26(3), 239-257.

Cattell, R. B. (1952). The three basic factor-analytic research designs-their interrelations and derivatives. Psychological Bulletin, 49(5), 499-520.

Chin, A., Markey, A., Bhargava, S., Kassam, K. S., \& Loewenstein, G. (2017). Bored in the USA: Experience Sampling and Boredom in Everyday Life. Emotion, 17(2), 359-368.

Cronbach, L. J. (1947). Test "reliability": Its meaning and determination. Psychometrika, 12(1), $1-16$.

Cronbach, L. J. (1951). Coefficient alpha and the internal structure of tests. Psychometrika, 16(3), 297-334.

de Vries, R. E., Realo, A., \& Allik, J. (2016). Using Personality Item Characteristics to Predict Single-Item Internal Reliability, Retest Reliability, and Self-Other Agreement. European Journal of Personality, 30(6), 618-636.

Denissen, J. J. A., Geenen, R., Selfhout, M., \& Van Aken, M. A. G. (2008). Single-item Big Five Ratings in a Social Network Design. European Journal of Personality, 22(1), 37-54.

Fisher, G. G., Matthews, R. A., \& Gibbons, A. M. (2015). Developing and Investigating the Use of Single-Item Measures in Organizational Research. Journal of Occupational Health Psychology, 21(1). 
Gulliksen, H. (1945). The Relation of Item Difficulty and Inter-item Correlation to Test Variance and Reliability. Psychometrika, 10(2), 79-91.

Guttman, L. (1945). A basis for analyzing test-retest reliability. Psychometrika, 10(4), 255-282.

John, O. P., \& Soto, C. J. (2007). The importance of being valid: Reliability and the process of construct validation. In R. W. Robins, R. C. Fraley, R. F. Krueger, R. W. (Ed) Robins, R. C. (Ed) Fraley, \& R. F. (Ed) Krueger (Eds.), Handbook of research methods in personality psychology. (pp. 461-494). New York, NY, US: Guilford Press.

Lord, F. M., \& Novick, M. R. (1968). Statistical theories of mental test scores. Reading, MA: Addison-Wesley.

McCrae, R. R., Kurtz, J. E., Yamagata, S., \& Terracciano, A. (2011). Internal consistency, retest reliability, and their implications for personality scale validity. Personality and Social Psychology Review, 15(1), 28-50.

Nunnally, J. C., \& Bernstein, I. H. (1991). Psychometric theory. New York, NY: McGraw.

Osburn, H. G. (2000). Coefficient alpha and related internal consistency reliability coefficients. Psychological Methods, 5(3), 343-355.

Soto, C. J., \& John, O. P. (2016). The next Big Five Inventory (BFI-2): Developing and assessing a hierarchical model with 15 facets to enhance bandwidth, fidelity, and predictive power. Journal of Personality and Social Psychology.

Spörrle, M., \& Bekk, M. (2014). Meta-analytic guidelines for evaluating single-item reliabilities of personality instruments. Assessment, 21(3), 272-282.

Vaidya, J. G., Gray, E. K., Haig, J. R., Mroczek, D. K., \& Watson, D. (2008). Differential Stability and Individual Growth Trajectories of Big Five and Affective Traits During Young Adulthood. Journal of Personality, 76(2), 267-304. 
Vaidya, J. G., Gray, E. K., Haig, J., \& Watson, D. (2002). On the temporal stability of personality: evidence for differential stability and the role of life experiences. Journal of Personality and Social Psychology, 83(6), 1469-1484.

Wanous, J. P., \& Hudy, M. J. (2001). Single-Item Reliability: A Replication and Extension. Organizational Research Methods, 4(4), 361-375.

Wanous, J. P., \& Reichers, A. E. (1996). Estimating the Reliability of a Single-Item Measure. Psychological Reports, 78, 631-634.

Watson, D., \& Clark, L. A. (1999). The PANAS-X: Manual for the positive and negative affect schedule-expanded form.

Watson, D., Hubbard, B., \& Wiese, D. (2000). Self-other agreement in personality and affectivity: The role of acquaintanceship, trait visibility, and assume similarity. Journal of Personality and Social Psychology, 78(3), 546-558.

Weidman, A. C., Steckler, C. M., \& Tracy, J. L. (2017). The jingle and jangle of emotion assessment: Imprecise measurement, casual scale usage, and conceptual fuzziness in emotion research. Emotion, 17(2), 267-295.

Weiss, D. J. (1976). Multivariate procedures. In M. D. Dunnette (Ed.), Handbook of Industrial and Organizational Psychology (pp. 327-362). Chicago, IL: Rand McNally College.

Wood, D., Harms, P. D., Lowman, G. H., \& Desimone, J. A. (2017). Response speed and response consistency as mutually validating indicators of data quality in online samples. Social Psychological and Personality Science.

Wood, D., Harms, P. D., Lowman, G. H., Soto, C., Lin, Q., John, O., \& Jiahui, L. (2017). Exploring the Utility of Within-Session Retest Correlations as Reliability Estimates. Tuscaloosa, AL. 
Wood, D., \& Wortman, J. (2012). Trait Means and Desirabilities as Artifactual and Real Sources of Differential Stability of Personality Traits. Journal of Personality, 80(3), 665-701. 


\section{Tables}

Table 1. PANAS-X Reliability Estimates

\begin{tabular}{|c|c|c|c|}
\hline Item & $\bar{M}_{W S}(\overline{S D})$ & $\overline{\boldsymbol{r}}_{\mathbf{X}(\boldsymbol{d})}(\overline{\boldsymbol{S} D})$ & $\widetilde{I C}_{x}(\overline{S D})$ \\
\hline Cheerful & $2.98(1.07)$ & $.79(.09)$ & $.66(.17)$ \\
\hline Disgusted & $1.49(.81)$ & $.59(.09)$ & $.45(.15)$ \\
\hline Attentive & $3.50(.92)$ & $.68(.08)$ & $.63(.02)$ \\
\hline Bashful & $1.63(.86)$ & $.71(.14)$ & $.46(.18)$ \\
\hline Sluggish & $2.04(1.04)$ & $.67(.13)$ & $.52(.10)$ \\
\hline Daring & $1.94(1.01)$ & $.67(.10)$ & $.41(.13)$ \\
\hline Surprised & $1.79(.90)$ & $.59(.03)$ & $.44(.16)$ \\
\hline Strong & $2.88(1.18)$ & $.80(.01)$ & $.62(.01)$ \\
\hline Scornful & $1.42(.77)$ & $.52(.13)$ & $.41(.18)$ \\
\hline Relaxed & $3.31(1.04)$ & $.70(.11)$ & $.72(.15)$ \\
\hline Irritable & $1.88(.93)$ & $.67(.07)$ & $.45(.22)$ \\
\hline Delighted & $2.53(1.17)$ & $.74(.05)$ & $.62(.09)$ \\
\hline Inspired & $2.66(1.19)$ & $.74(.02)$ & \\
\hline Fearless & $2.15(1.12)$ & $.64(.06)$ & $.47(.09)$ \\
\hline Disgusted with self & $1.55(.89)$ & $.70(.14)$ & $.73(.12)$ \\
\hline Sad & $1.72(.93)$ & $.71(.11)$ & $.59(.10)$ \\
\hline Calm & $3.43(1.04)$ & $.67(.04)$ & $.70(.10)$ \\
\hline Afraid & $1.46(.77)$ & $.62(.04)$ & $.61(.06)$ \\
\hline Tired & $2.47(1.13)$ & $.73(.09)$ & $.65(.13)$ \\
\hline Amazed & $1.94(1.08)$ & $.72(.05)$ & $.64(.18)$ \\
\hline Shaky & $1.41(.72)$ & $.77(.05)$ & $.41(.12)$ \\
\hline Happy & $3.14(1.12)$ & $.78(.04)$ & $.70(.05)$ \\
\hline Timid & $1.70(.91)$ & $.71(.10)$ & $.76(.08)$ \\
\hline Alone & $1.90(1.13)$ & $.84(.03)$ & $.61(.03)$ \\
\hline Alert & $3.20(1.09)$ & $.72(.06)$ & $.57(.11)$ \\
\hline Upset & $1.66(.88)$ & $.69(.09)$ & \\
\hline Angry & $1.53(.79)$ & $.64(.05)$ & $.61(.06)$ \\
\hline Bold & $2.13(1.07)$ & $.75(.04)$ & $.57(.08)$ \\
\hline Blue & $1.77(.99)$ & $.80(.10)$ & $.65(.16)$ \\
\hline Shy & $1.86(1.02)$ & $.77(.08)$ & $.52(.15)$ \\
\hline Active & $2.89(1.15)$ & $.74(.04)$ & \\
\hline Guilty & $1.45(.74)$ & $.71(.11)$ & $.51(.02)$ \\
\hline Joyful & $2.64(1.21)$ & $.81(.05)$ & $.76(.01)$ \\
\hline
\end{tabular}

Notes. Average $\bar{r}_{\mathrm{X}(\mathrm{d})}$ estimates were calculated by taking the average of within-session (ws) item correlations across $\mathrm{MT}_{1}, \mathrm{MT}_{2}$, and SS samples. Average $\widetilde{I C}_{x}$ estimates were calculated by taking the average item communality based on a principal axis factor analysis of the first administration across the three within-session samples. Blank cells indicate that $\widetilde{I C}_{x}$ was not calculated for this item, for reasons described in text. Corrections for attention for test-retest correlations over 1 week, 3 years, 6 years, 9 years, 13 years, as well as the self-other agreement correlations for the married, dating, and friends samples are provided in the Supplementary Materials. 
Table 1. PANAS-X Reliability Estimates (continued)

\begin{tabular}{lccc} 
& $\overline{\boldsymbol{M}}_{\boldsymbol{W S}}(\overline{\boldsymbol{S D}})$ & $\overline{\boldsymbol{r}}_{\mathbf{X}(\boldsymbol{d})}(\overline{\boldsymbol{S D}})$ & $\widetilde{\boldsymbol{I C}}_{\boldsymbol{x}}(\overline{\boldsymbol{S D}})$ \\
\hline Nervous & $1.79(.92)$ & $.68(.00)$ & $.52(.07)$ \\
Lonely & $1.83(1.11)$ & $.85(.05)$ & $.63(.07)$ \\
Sleepy & $2.39(1.11)$ & $.77(.08)$ & $.79(.17)$ \\
Excited & $2.44(1.10)$ & $.73(.01)$ & $.60(.05)$ \\
Hostile & $1.40(.75)$ & $.56(.00)$ & $.49(.03)$ \\
Proud & $2.46(1.19)$ & $.80(.04)$ & $.40(.12)$ \\
Jittery & $1.50(.75)$ & $.70(.04)$ & $.42(.06)$ \\
Lively & $2.58(1.12)$ & $.71(.05)$ & $.66(.07)$ \\
Ashamed & $1.36(.70)$ & $.62(.15)$ & $.53(.21)$ \\
At ease & $3.14(1.18)$ & $.71(.01)$ & $.66(.09)$ \\
Scared & $1.41(.72)$ & $.70(.13)$ & $.66(.03)$ \\
Drowsy & $2.12(1.09)$ & $.75(.07)$ & $.69(.07)$ \\
Angry at self & $1.55(.86)$ & $.67(.11)$ & $.70(.12)$ \\
Enthusiastic & $2.72(1.18)$ & $.78(.04)$ & $.71(.05)$ \\
Downhearted & $1.76(.97)$ & $.72(.05)$ & $.63(.00)$ \\
Sheepish & $1.49(.82)$ & $.65(.14)$ & $.41(.07)$ \\
Distressed & $1.63(.88)$ & $.75(.02)$ & $.55(.14)$ \\
Blameworthy & $1.43(.74)$ & $.68(.07)$ & $.38(.08)$ \\
Determined & $3.23(1.11)$ & $.71(.07)$ & $.61(.12)$ \\
Frightened & $1.38(.65)$ & $.69(.03)$ & $.53(.06)$ \\
Astonished & $1.63(.86)$ & $.68(.04)$ & $.47(.08)$ \\
Interested & $3.14(1.07)$ & $.65(.05)$ & $.56(.06)$ \\
Loathing & $1.41(.77)$ & $.63(.07)$ & $.61(.05)$ \\
Confident & $2.97(1.18)$ & $.79(.08)$ & $.44(.16)$ \\
Energetic & $2.68(1.15)$ & $.73(.04)$ & $.64(.06)$ \\
Concentrating & $3.27(.98)$ & $.68(.06)$ & $.58(.10)$ \\
Dissatisfied with self & $1.66(.95)$ & $.59(.15)$ & 18 \\
Average Estimate & & & \\
Corrections for Attenuation & & $.71(.07)$ & 0 \\
\hline
\end{tabular}

Notes. Average $\bar{r}_{\mathrm{X}(\mathrm{d})}$ estimates were calculated by taking the average of within-session (ws) item correlations across $\mathrm{MT}_{1}, \mathrm{MT}_{2}$, and SS samples. Average $\widetilde{I C}_{x}$ estimates were calculated by taking the average item communality based on a principal axis factor analysis of the first administration across the three within-session samples. Blank cells indicate that $\widetilde{I C}_{x}$ was not calculated for this item, for reasons described in text. Corrections for attention for test-retest correlations over 1 week, 3 years, 6 years, 9 years, 13 years, as well as the self-other agreement correlations for the married, dating, and friends samples are provided in the Supplementary Materials. 
Table 2. Between-Item Associations, Within-Session Retest Correlations and PANAS-X Item Properties

\begin{tabular}{|c|c|c|c|c|c|c|c|c|c|c|c|c|c|c|c|c|c|}
\hline Item Property & $M(S D)$ & $\widetilde{I C}_{X}$ & $\overline{\boldsymbol{r}}_{X(d)}$ & $\mathbf{M T}_{1}$ & $\mathbf{M T}_{2}$ & SS & Item $_{M}$ & Item $_{\text {Dev }}$ & Item $_{S D}$ & $r_{\mathrm{X}(\text { avg.6-7) }}$ & $r \mathbf{X}(3 \mathrm{yr})$ & $r_{\mathbf{x}(6 \mathrm{yr})}$ & $r_{\mathrm{X}}(9 \mathrm{yr})$ & $r \mathrm{X}(13 \mathrm{yr})$ & $r_{\text {so(Married) }}$ & $r_{\text {so(dat }}$ & ting) \\
\hline Approx. internal consistency; $\widetilde{I C}_{X}$ & $.58(.11)$ & -- & & & & & & & & & & & & & & & \\
\hline Average within-session retest corr.; $\bar{r}_{X(d)}$ & $.71(.07)$ &.$\underline{.39}$ & -- & & & & & & & & & & & & & & \\
\hline MTurk Sample $1 r_{X(d)} ; \mathrm{MT}_{1}$ & $.70(.07)$ & .33 & .80 & -- & & & & & & & & & & & & & \\
\hline MTurk Sample $2 r_{X(d)} ; \mathrm{MT}_{2}$ & $.75(.10)$ &. .37 & .78 & .45 & -- & & & & & & & & & & & & \\
\hline Student Sample $1 r_{X(d)} ; \mathrm{SS}$ & $.67(.09)$ & .21 &.$\underline{.75}$ & $\underline{.48}$ & .30 & -- & & & & & & & & & & & \\
\hline \multicolumn{18}{|l|}{ Additional Item Properties } \\
\hline Item mean; Item $_{M}$ & $2.14(.66)$ & .29 & $\underline{.35}$ & $\underline{.44}$ & .19 & .23 & -- & & & & & & & & & & \\
\hline Item deviance from scale midpoint; Item $_{D e v}$ & $.94(.54)$ & -.31 & $\underline{-.46}$ & $\underline{-.49}$ & -.26 & $\underline{-.35}$ & $\underline{-.96}$ & -- & & & & & & & & & \\
\hline Item standard deviation; Item $S D$ & $.98(.16)$ & $\underline{.35}$ & $\underline{.60} \mathrm{a}$ & $\underline{.52}$ & $\underline{.35}$ & $\underline{.55}$ & $\underline{.75}$ & $\underline{-.86}$ & -- & & & & & & & & \\
\hline \multicolumn{18}{|l|}{ Validity-Related Criteria } \\
\hline Retest correlation, 1 week; $r_{X(\text { avg.6-7) }}$ & $.32(.11)$ & $-.04 \mathrm{a}$ & $\underline{.33}$ a & $\underline{.37}$ & .17 & .26 & .31 &.- .33 & .31 & -- & & & & & & & \\
\hline Retest correlation, 3 years; $r_{X(3 y r)}$ & $.37(.06)$ & .16 & .29 & .25 & .16 & .29 & .07 & -.14 & $\underline{.35}$ & .18 & -- & & & & & & \\
\hline Retest correlation, 6 years; $r_{X(6 y r)}$ & $.32(.07)$ & -.09 & .05 & .07 & -.04 & .10 & -.05 & -.01 & .08 & .07 & $\underline{.64}$ & -- & & & & & \\
\hline Retest correlation, 9 years; $r_{X(9 \mathrm{yr})}$ & $.28(.07)$ & .11 & .02 & .15 & -.01 & -.05 & .15 & -.20 & .19 & .05 & .41 & $\underline{.47}$ & & -- & & & \\
\hline Retest correlation, 13 years; $r_{X(13 \mathrm{yr})}$ & $.26(.07)$ & -.32 & $-.01_{a}$ & -.02 & -.05 & .06 & -.02 & -.03 & .01 & .09 & .32 &.$\underline{.42}$ & & .40 & -- & & \\
\hline Self-other agreement, Married; $r_{\mathrm{so} \text { (married) }}$ & $.29(.13)$ & .30 & .19 & .09 & .14 & .18 & -.02 & -.08 & .20 & .26 & .32 & .25 & & .42 & .02 & -- & \\
\hline Self-other agreement, dating; $r_{\mathrm{so} \text { (dating) }}$ & $.19(.08)$ & $-.04 \mathrm{a}$ & $.30_{\mathrm{a}}$ & .34 & .11 & .27 & .12 & -.21 & .36 & $\underline{.38}$ & $\underline{.51}$ & $\underline{.38}$ & & .32 & .26 & $\underline{.40}$ & -- \\
\hline Self-other agreement, friends; $r_{\mathrm{so} \text { (friends) }}$ & $.19(.08)$ & .14 & .37 & $\underline{.45}$ & .11 & .36 & .27 &.- .34 & .41 & .25 & .57 & $\underline{.35}$ & & .33 & .19 & .28 &. \\
\hline
\end{tabular}

Notes. $N=55$ for all correlations involving $\widetilde{I C}_{X}$ estimates; $N=60$ for all other cells. Bolded values indicate $p<.05$; bolded and underlined values indicate $p<.01$.

$\widetilde{I C}_{X}$ indicate average item-communality (or squared factor loading) when items from a subscale are used in principal axis factoring, as described in text. $r_{X}(t)=$ retest correlation for item X over interval $t . r_{s o(p)}=$ self-other correlation between type of partner $p$ given in parentheses. Correlations involving 3-sample average within-session retest correlations, $\bar{r}_{x(d)}$, are italicized. Remaining column abbreviations are expanded in row headings. Subscript a indicates that correlates of $\widetilde{I C}_{X}$ and $r_{X(d)}$ estimates of reliability differ significantly by Steiger's (1980) test of differences in dependent correlations. 